\title{
WDM Orthogonal Subcarrier Multiplexing
}

\author{
Fernando A. Gutiérrez, Eamonn P. Martin, Philip Perry, Andrew D. Ellis, Member, IEEE, Prince M. \\ Anandarajah, Senior Member, IEEE, and Liam P. Barry, Senior Member, IEEE.
}

\begin{abstract}
Electro-optical transceivers can be implemented employing all-analogue signal processing in order to achieve low values of power consumption and latency. This paper shows that the spectral efficiency of such solutions can be increased by combining orthogonal multicarrier techniques and off-the-shelf microwave components. A real-time $108 \mathrm{Gbit} / \mathrm{s}$ experiment was performed emulating a wavelength division multiplexing (WDM) system composed of five optical channels. The optical carriers were provided by an externally injected gain switched optical frequency comb. Each optical channel transmitted a $21.6 \mathrm{Gbit} / \mathrm{s}$ orthogonal subcarrier multiplexing (SCM) signal that was modulated and demodulated in the electrical domain without the requirement for digital signal processing. The net data rate remained higher than $100 \mathrm{Gbit} / \mathrm{s}$ after taking into account forward error correction overheads. The use of orthogonally overlapping subchannels achieves an unprecedented spectral efficiency in all-analogue real-time broadband WDM/SCM links.
\end{abstract}

Index Terms - Electro-optical transceiver, Filter Bank Multicarrier (FBMC), Orthogonal Frequency Division Multiplexing (OFDM), Subcarrier Multiplexing (SCM).

\section{INTRODUCTION}

$\mathrm{T}$ HE increasing demand for capacity in optical networks has translated into a growing interest and research effort in high-speed short-reach optical interconnects and its associated technologies [1]. For practical and real-time applications, electro-optical transceivers are preferred due to the stability and maturity of electronic circuits and microwave components, in contrast with their optical counterparts. The broad range of possible electronic implementations presents a trade-off between three major figures of merit in communications systems: spectral efficiency, power consumption and latency. In recent years, the majority of advances in the area have been supported by digital signal processing (DSP) due to its ability to increase spectral efficiency and mitigate impairments [2]. However, a high number of computational operations also brings about disadvantages such as increased power consumption [3] and often latency. This fact motivates the investigation of electro-

Manuscript submitted on October 9, 2015. This work was supported in part by the EI CFTD grant CF/2011/1627, SFI grants 12/RC/2276, 10/CE/I1853 and 14/TIDA/2405 and EPSRC grant EP/L000091/1.

F. A. Gutierrez, E. P. Martin, P. Perry, P. M. Anandarajah and L. P. Barry are with The RINCE Institute, Dublin City University, Glasnevin, Dublin 9, Ireland. (E-mail: fernando.gutierrez@dcu.ie).

A. D. Ellis is with Aston Institute of Photonic Technologies, Aston University, Aston Triangle, Birmingham B4 7ET, UK.

Copyright (c) 2015 IEEE. Personal use of this material is permitted. However, permission to use this material for any other purposes must be obtained from the IEEE by sending a request to pubs-permissions@ieee.org. optical transceivers that rely on all-analogue signal processing to maximize power efficiency [4] and minimize latency.

Nowadays, there is a proliferation of data centers addressing a variety of services. Most data center subsystems need moderate total capacity but a high capacity per interface while reducing power consumption and latency to a minimum. For such applications, a purely analogue implementation potentially presents the best trade-off between those key parameters. Besides, there are other important requirements which determine the most appropriate transmission scheme. Firstly, tolerance to dispersion is required to have compatibility between inter and intra data center distances. Secondly, the solution must be compatible with electrical interfaces that consist of digital data lines at Gbit/s, simplifying the buffering and (de)multiplexing of data at different speeds. Finally, integration with wavelength division multiplexing (WDM) systems must be feasible. Subcarrier multiplexing (SCM) is one potential solution that meets all the requirements. SCM generates a multicarrier electrical signal that is then transmitted over fibre on an optical carrier [5]. A purely analogue SCM optical single side band (OSSB) scheme is possible and compatible with the desired baseband rates using microwave low pass filters (LPF), mixers, combiners and splitters [6]. Moreover, the overall electrical transceiver can be integrated using monolithic microwave integrated circuit (MMIC) technology [7]. High tolerance to dispersion is achieved with the OSSB modulation [8], while allowing a closer allocation of optical subchannels in WDM systems [9]. An optimum optical transmitter to provide such closely spaced optical subchannels must rely on an optical frequency comb (OFC) source. In contrast with independent lasers, an OFC offers constant frequency spacing between the carriers. The main weakness of all-analogue SCM is the spectral efficiency, but it can be substantially improved by employing orthogonally overlapping subchannels according to filter bank multicarrier (FBMC) theory [10, 11]. An optical link based on this concept will be referred to as orthogonal SCM (OSCM) in the rest of the text.

This paper presents a direct detection (DD) 5x21.6 Gbit/s real-time all-analogue WDM/OSCM transmission scheme. The modulation and demodulation of the orthogonal data is achieved using off-the-shelf microwave components. The WDM system is emulated employing an externally injected gain switched OFC [12]. Performance is measured and compared with a single optical channel implementation. The net rate after applying a forward error correction (FEC) code with a $7 \%$ overhead would still be higher than $100 \mathrm{Gbit} / \mathrm{s}$. The achieved spectral efficiency is higher than in any previously 


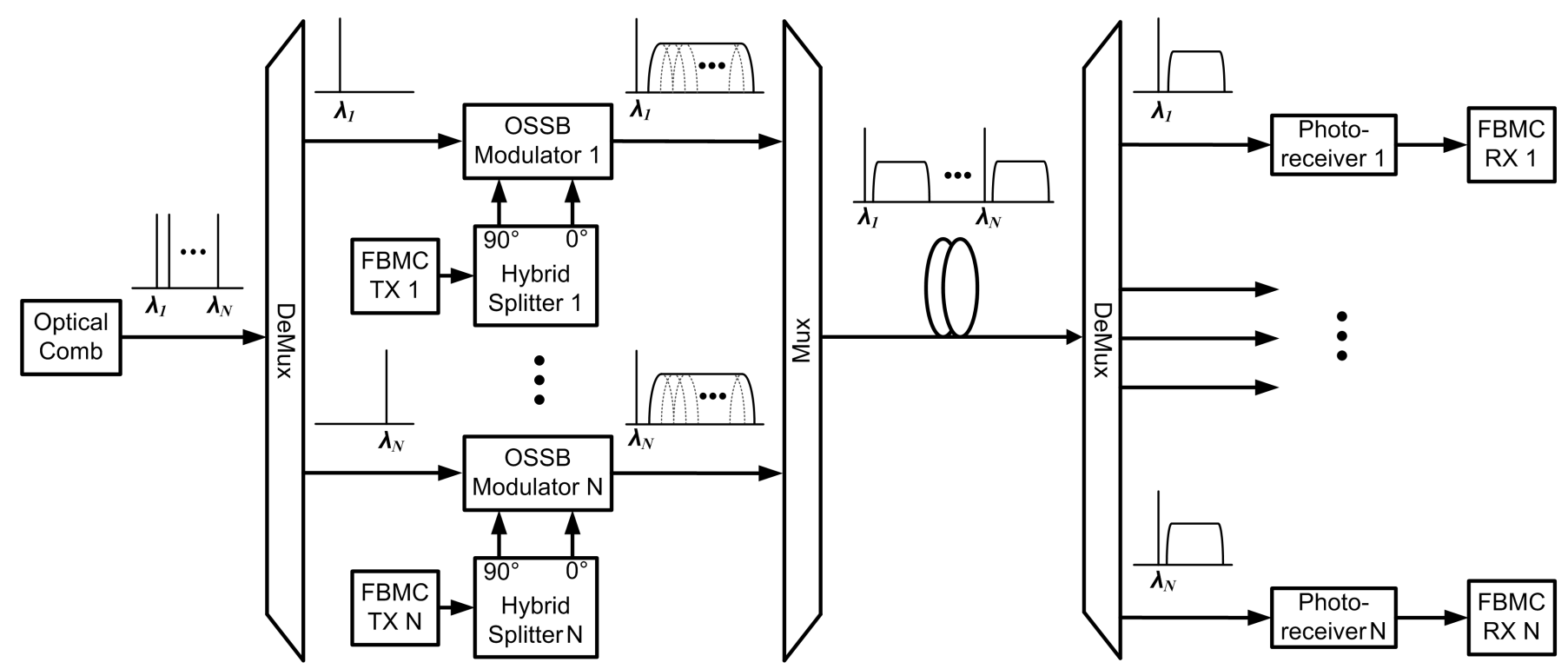

Fig. 1. WDM/OSCM/OSSB transmission scheme with $N$ optical subchannels and based on an OFC.

reported DSP-less broadband WDM/SCM system.

\section{WDM/OSCM}

This section discusses a generic WDM/OSCM system and describes the major electrical and optical concepts focusing on a physical implementation.

\section{A. Generic WDM/OSCM Scheme}

A WDM/OSCM/OSSB transmission scheme composed of $N$ optical subchannels and based on an OFC is shown in Fig. 1. The use of an OFC instead of individual lasers reduces the number of required components and ensures the maintenance of constant frequency spacing between the optical channels. The optical carriers at the output of the OFC are demultiplexed and each one is modulated producing an OSSB signal. This can be achieved with dual drive Mach Zehnder (DD-MZM) modulators [8] or with optical IQ modulators (OIQM) [13] whose electrical inputs are fed with the desired signal and its Hilbert transformed (HT) pair. The HT of an electrical signal can be obtained directly in the analogue domain by employing a microwave hybrid splitter. The modulating electrical signal is generated with an all-analogue FBMC transmitter and is composed of orthogonally overlapping subchannels carrying digital data. The $N$ optical channels are combined and transmitted over fibre. In the receiver, the optical channels are demultiplexed and DD is used to recover the FBMC electrical signals. Finally, allanalogue FBMC receivers demodulate the orthogonal electrical subchannels. The next subsection explains the realization of the all-analogue FBMC blocks.

\section{B. Microwave FBMC}

The FBMC technique was initially defined in [14]. The technique has been developed for decades, but often focusing on DSP based implementations [15]. The suitability of FBMC for wired and optical fibre communications has been stated theoretically [15] and experimentally [16]. According to
FBMC, the transmission of orthogonal subchannels carrying digital data can be accomplished without requiring the fast Fourier transform (FFT). The following three conditions must be ensured. Firstly, every pair of baseband digital streams, which will modulate a particular subcarrier, must present a relative time shift equal to half a bit period. Secondly, the delayed and non-delayed baseband streams that form a pair must act as the in-phase and the quadrature components alternately in different subcarriers. Finally, baseband bits at the transmitter must be shaped as square root raised cosine (SRRC) pulses, and the baseband filter at the receiver must present the matched SRRC response. Meeting the previous conditions, each received baseband signal will present a sampling point in the middle of the bit period that will be free of inter symbol and inter channel interference (ISI and ICI).

Fig. 2 shows a back to back microwave FBMC scheme consisting of three orthogonal subchannels. The conditions explained in the previous paragraph can be found alongside other parameters that are present due to the purely analogue implementation. Firstly, the digital data is sourced with a square shape. For a baseband rate of $r \mathrm{bit} / \mathrm{s}$, the baseband spectrum is a sinc function, $\operatorname{SINC}_{r}(f)$. Secondly, different microwave tracks and IQ mixers can present totally different delays. However, perfect orthogonal phase alignment in the transmitter is still possible by including extra phase shifts in the local oscillators (LO), $\Delta \phi_{i}[11]$. These phase shifts ensure that ICI cancels at the sampling point where ISI is zero. For simplicity, the phases of the LOs in the receiver are the same, considering that there are no additional delays after the IQ mixers in the transmitter. As in any frequency division multiplexing scheme, a phase locked loop (PLL) must be used in the receiver to achieve phase and frequency locking [17, 18]. Finally, the low pass filters in the transmitter and the receiver, $L P F_{T X}(f)$ and $L P F_{R X}(f)$, must be sinc compensated SRRC and standard SRRC respectively. That is the only set of filters which would guarantee perfect functionality, free of ISI and ICI. The eye diagrams in Fig. 2 show the received signals 


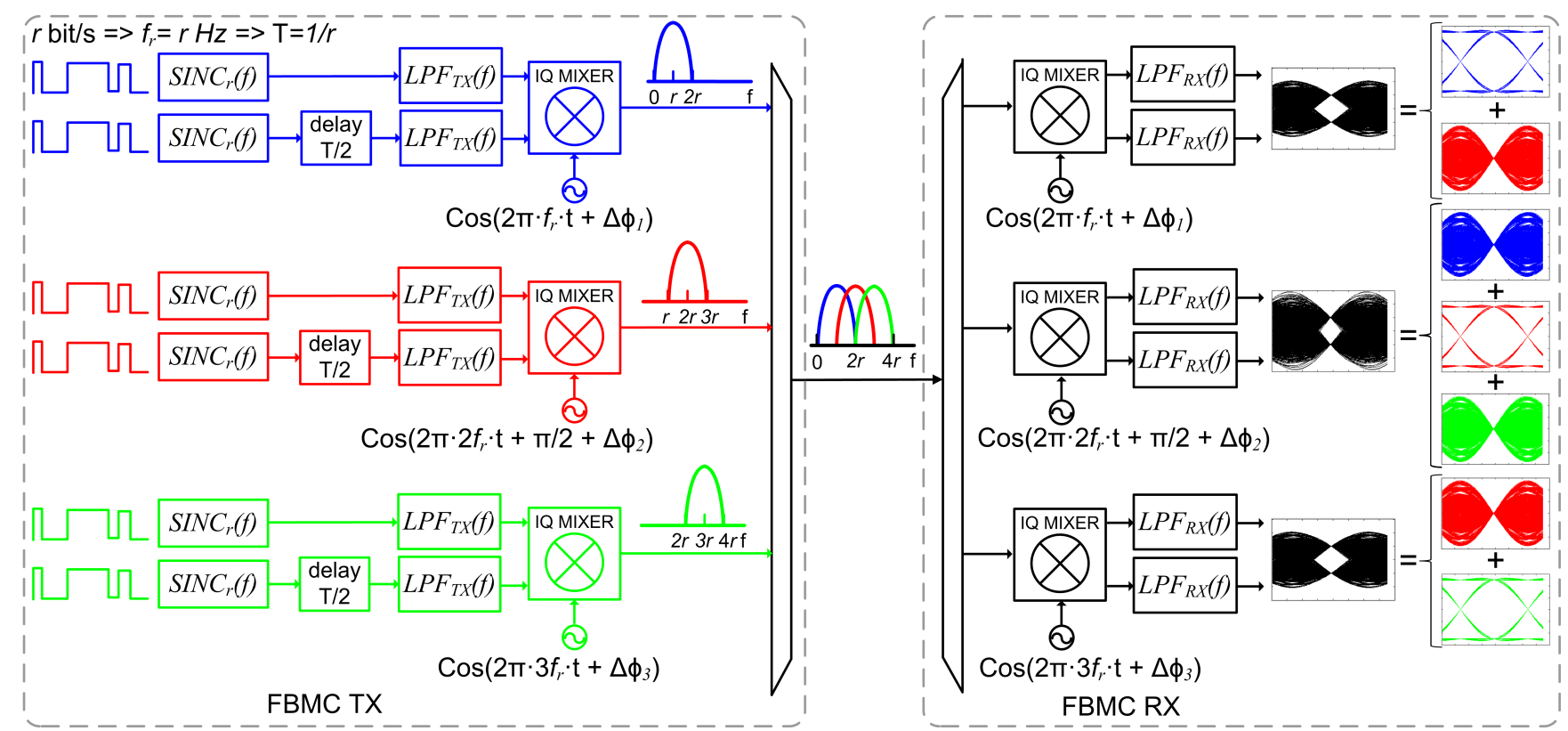

Fig. 2. Back to back microwave FBMC scheme consisting of 3 orthogonally overlapping subchannels.

when perfect SRRC filters with roll-off factor $\beta$ equal to 1 are used. Each channel in the transmitter is associated with a different color. The contribution of each transmitted subchannel in the received signals is illustrated using the corresponding color.

As the SRRC filters are difficult to design in the microwave domain, a solution based on off-the-shelf filters was investigated. The baseband transfer functions of the transmitter, receiver and transmitter plus receiver are represented by $H_{T X}(f), H_{R X}(f)$, and $H_{T R X}(f)$ respectively:

$$
\begin{aligned}
& H_{T X}(f)=\operatorname{SINC}_{r}(f) \cdot \operatorname{LPF}_{T X}(f) . \\
& H_{R X}(f)=L P F_{R X}(f) . \\
& H_{T R X}(f)=\operatorname{SINC}_{r}(f) \cdot \operatorname{LPF}_{T X}(f) \cdot \operatorname{LPF}_{R X}(f) .
\end{aligned}
$$

Assuming that the LPFs have constant group delay, perfect ISI and ICI cancellation are achieved when the following conditions are met [14]:

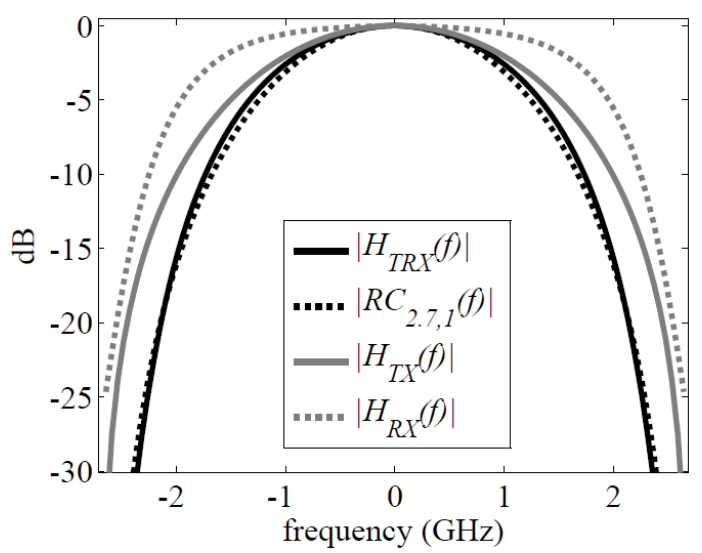

Fig. 3. Baseband transfer functions using two cascaded Bessel filters $\left(B_{2,4}(f)\right)$ in the receiver for a rate $r=2.7 \mathrm{Gbit} / \mathrm{s}$.

$$
\begin{aligned}
& I S I=0 \rightarrow\left|H_{T R X}(f)\right| \propto\left|R C_{r, \beta}(f)\right| . \\
& I C I=0 \rightarrow\left|H_{T X}(f)\right| \propto\left|H_{R X}(f)\right| .
\end{aligned}
$$

Where $R C_{r, \beta}(f)$ represents the raised cosine spectra of rate $r$ $\mathrm{Gbit} / \mathrm{s}$ and roll-off factor $\beta(0 \leq \beta \leq 1)$. Typically, microwave wired communications links make use of Bessel filters (of order 4) because of the maximally flat group delay. The transfer function of a Bessel filter with a $3 \mathrm{~dB}$ cut-off frequency of $f_{c} \mathrm{GHz}$ and order $n$ is denoted as $B_{f c, n}(f)$. A solution based on this type of filters was investigated. For a baseband rate, $r$, of $2.7 \mathrm{Gbit} / \mathrm{s}$, the proposed solution consists of using a filter that passes the first lobe of the sinc in the transmitter and two cascaded $B_{2,4}(f)$ filters in the receiver:

$$
\begin{aligned}
& \left|H_{T X}(f)\right|=\left|\operatorname{SINC}_{2.7}(f)\right| . \\
& H_{R X}(f)|=| B_{2,4}(f) \cdot B_{2,4}(f) \mid . \\
& \left|H_{T R X}(f)\right|=\left|\operatorname{SINC}_{2.7}(f) \cdot B_{2,4}(f) \cdot B_{2,4}(f)\right| .
\end{aligned}
$$

Fig. 3 illustrates the previous transfer functions and analyzes them following the conditions stated in eq. (2). The overall

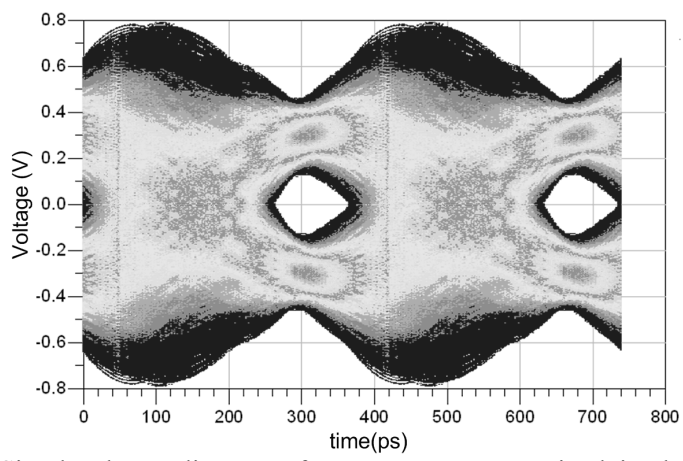

Fig. 4. Simulated eye diagram of one component received in the middle subchannel of a back to back three subcarrier FBMC system implemented with off-the-shelf filters. PRBS15 nattern was emnloved for this simulation. 
transfer function $H_{T R X}(f)$ is similar to a raised cosine spectrum $R C_{2.7,1}(f)$. In contrast, the baseband transfer functions in the transmitter and the receiver are different. It can be concluded that good ISI cancelation would be achieved while the main source of distortion would be generated by ICI. To test the overall performance of the solution, a simulation of the scheme presented in Fig. 2 was performed using the software ADS. IQ mixers were considered to be ideal and pseudo random binary sequences (PRBS) of $2^{15}-1$ bits were employed. The LPFs in the receiver were the two cascaded $B_{2,4}(f)$. In the transmitter, there are many filters that could be used, as the proposed solution relies on passing the first lobe of the sinc. For consistency with the experimental results presented in section III, the simulation made use of the filter Minicircuits model VLF-2500. This filter has the $3 \mathrm{~dB}$ cut-off frequency at $3.075 \mathrm{GHz}$ and a group delay ripple of $200 \mathrm{ps}$ till $2.5 \mathrm{GHz}$. The received eye diagram for one component of the middle subchannel is shown in Fig. 4. Despite the impairments, the eye is open confirming the viability of the solution. It should be noted that the use of Bessel filters in the transmitter (namely $B_{4,4}(f)$ ) was also tested achieving similar performance.

Unlike FFT-based orthogonal frequency division multiplexing (OFDM) [19], with FBMC the dispersion suffered by any electrical subchannel will only influence the performance of its adjacent subchannels. For low transmission distances, this penalty would be negligible while the absence of a cyclic prefix maximizes the data rate [14].

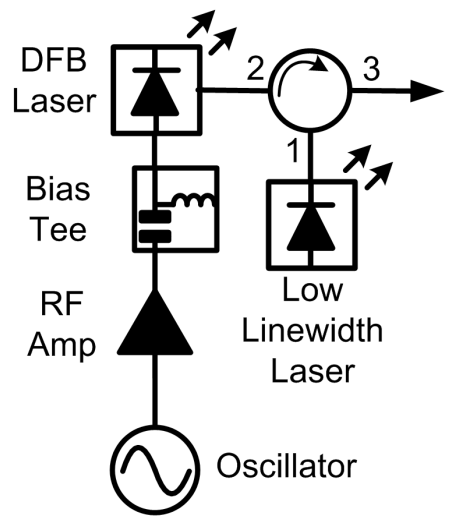

Fig. 5. Set-up for an OFC based on an externally injected gain-switched laser.

\section{Externally injected gain switched OFC}

Unlike independent lasers, OFCs offer constant frequency spacing between the optical carriers, allowing the reduction of inter-channel guard bands and the spacing between optical channels. However, an OFC needs to exhibit excellent spectral flatness, high frequency stability, low linewidth, high carrierto-noise ratio (CNR) and a tunable free spectral range (FSR), in order to be successfully employed in high capacity dense WDM systems. Conventional comb sources are mainly based on mode-locked semiconductor and fibre lasers [20,21]. Both techniques suffer from complex fabrication processes, a fixed FSR and stability issues associated with long cavity lengths (for the mode-locked fibre laser). An alternative technique, which offers the advantage of tunable FSR, entails the use of electro-optic modulators. This, however, requires the use of a series of cascaded modulators [22] or dual drive modulators
[23], in order to achieve a broad and flat comb. Inherent modulator properties such as bias drift and high insertion loss make this technique less attractive for network deployment.

OFC generation by gain switching of an externally injected distributed feedback (DFB) laser diode is an attractive alternative as it is a simple and cost efficient technique [24]. External injection offers beneficial properties to the OFC, such as an increase in the number of comb tones, improved spectral flatness, enhanced CNR, reduced relative intensity noise (RIN) and a reduced optical linewidth on each of the comb tones [25]. A typical set-up diagram of an OFC generated with an externally injected gain-switched DFB can be observed in Fig. 5. For DD systems, the performance of different optical channels depends on the RIN and the CNR of the optical lines in the OFC, as it has been demonstrated for OFDM signals [26].

\section{Optical link performance}

The performance of the individual electrical subchannels is typically measured by obtaining the sensitivity of the optical link, defined as the average optical power at the input of the optical receiver required to achieve a determined bit error rate (BER). Considering a hard-decision FEC (HD-FEC) code with a $7 \%$ overhead, the threshold of acceptable BER is $3.8 \cdot 10^{-3}$ [27]. A HD-FEC is compatible with the all-analogue nature of this approach as it corrects from ' 0 's and ' 1 's, in contrast with soft-decision FEC codes that require intermediate values in the input of the decoder.

The model presented in [13] calculates the lowest sensitivity that is theoretically achievable in an SCM/OSSB link based on an OIQM and a pre-amplified optical receiver. That sensitivity depends on the frequency plan, the modulation order, the symbol rate, the optical modulation index (OMI), the bias point of the OIQM and the noise figure of the optical amplifier. As explained in the subsequent section, the experiments consisted of four orthogonal 2.7 Gbaud quaternary phase shift keyed (QPSK) electrical subchannels located between the second and fifth harmonic of the baseband data rate. With that frequency plan and considering an Erbium doped fiber amplifier (EDFA) with a noise figure equal to $5 \mathrm{~dB}$, the estimated sensitivity was $\approx-28.2 \mathrm{dBm}$ for a total $\mathrm{rms}$ OMI of $20 \%$ with respect to the $V_{\pi}$ of the OIQM. The previous estimation also considered that the OIQM was biased at quadrature. When using orthogonal subchannels, this estimation is accurate only when the non-linear distortion generated by the OIQM is small compared with the optical noise. As this sensitivity was calculated for a high value of BER, $3.8 \cdot 10^{-3}$, or a high value of optical noise, a good agreement with experimental results was expected.

\section{EXPERIMENTS}

This section describes the physical link that was implemented to prove the feasibility of WDM/OSCM, developing the concepts that have been explained in section II with an experimental perspective.

\section{A. Experimental Setup}

A real-time DD WDM/OSCM system equivalent to that shown in Fig. 1 was emulated for the case of five optical 


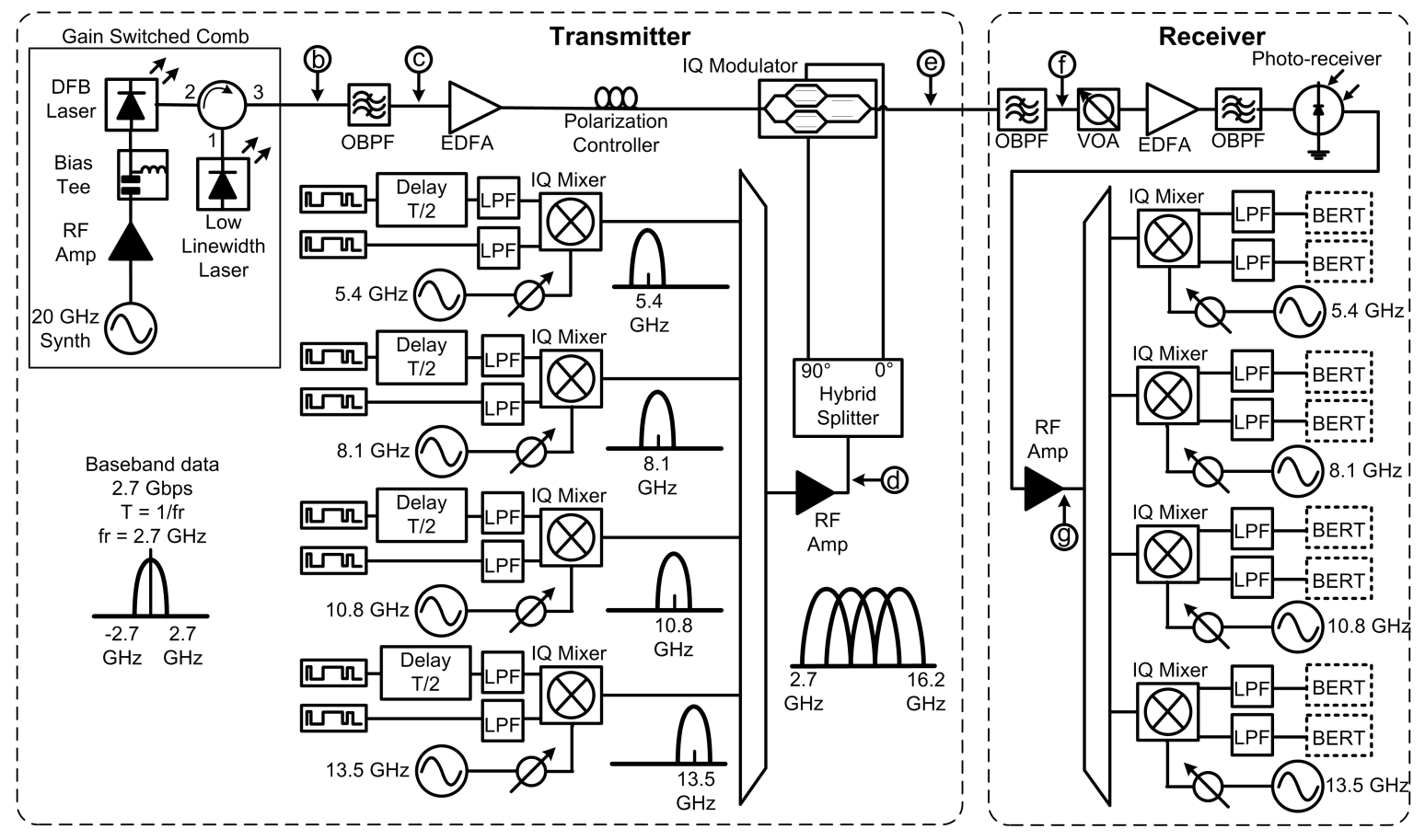

(a)

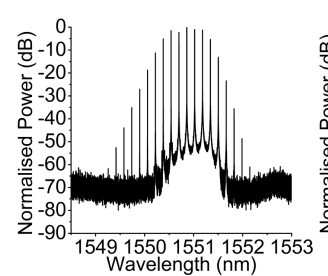

(b)

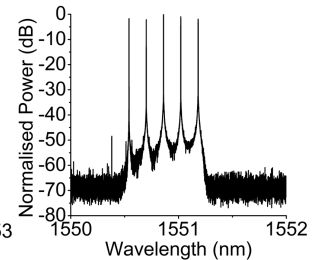

(c)

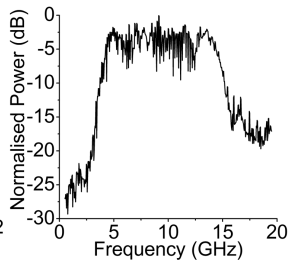

(d)

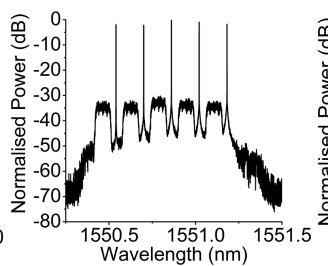

(e)

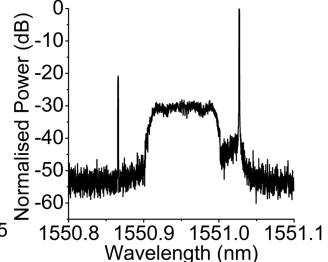

(f)

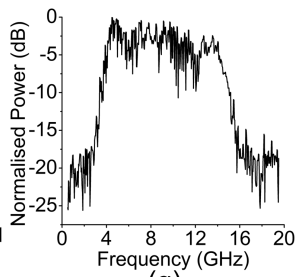

$(\mathrm{g})$

Fig. 6. (a) DD WDM/OSCM/OSSB link consisting of five optical channels with four orthogonal 2.7 Gbaud QPSK subcarriers each. Optical and electrical spectra are shown at: (b) the output of the optical comb, (c) the output of the optical filter that selects the optical lines that will be modulated, (d) the output of the RF FBMC transmitter, (e) the output of the optical modulator, (f) the output of the optical filter that selects one subchannel in the receiver, and (g) the output of the photo-receiver. The resolution of the optical spectra is $100 \mathrm{MHz}$.

channels separated by $20 \mathrm{GHz}$. The physical implementation, which made use of standard or off-the-shelf microwave components, is shown in Fig. 6(a). Inexpensive MMIC IQ mixers were employed to modulate and demodulate an FBMC signal consisting of four orthogonal 2.7 Gbaud offset-QPSK subchannels. An electrical comb generated the orthogonal LOs from a master reference of $2.7 \mathrm{GHz}$. A microwave filter demultiplexed the individual tones between the second and the fifth harmonics of the data rate $(5.4,8.1,10.8$ and $13.5 \mathrm{GHz})$. The described frequency plan produced an overall data rate of $21.6 \mathrm{Gbit} / \mathrm{s}$ over a bandwidth of $16.2 \mathrm{GHz}(1.333 \mathrm{bit} / \mathrm{s} / \mathrm{Hz})$ including the optical carrier or $13.5 \mathrm{GHz}(1.6 \mathrm{bit} / \mathrm{s} / \mathrm{Hz})$ excluding the optical carrier.

The OFC was generated with an externally injected gainswitched DFB laser, as explained in section II.C. The spectrum of the OFC is illustrated in Fig. 6(b). The FSR was $20 \mathrm{GHz}$, set by the radio frequency synthesizer used to drive the laser. Five lines of similar amplitude $(\approx 3 \mathrm{~dB}$ flatness $)$ were filtered, as can be observed in Fig. 6(c), and amplified. During the experiments, the OFC was also substituted with a highperformance external cavity laser (ECL) to measure the performance of the system in a single optical carrier case.
The FBMC transmitter was designed according to Fig. 2. A field programmable gate-array (FPGA) that integrated analogue transceivers was used to source eight bit streams at an individual rate of $2.7 \mathrm{Gbit} / \mathrm{s}$. The FPGA data clock was frequency locked to the master reference. Off-the-shelf MMIC IQ mixers are designed for radio applications, where lower data rate signals are employed. For that reason, they present an unwanted ripple of the group delay affecting performance when long binary sequences are transmitted [28]. To cancel this effect, short PRBS of $2^{7}-1$ bits were used. Each sequence was sourced by a separate pattern generator starting with a random offset. The $T / 2$ relative phase shift required in every pair of baseband signals was introduced using adjustable phase trimmers. Subsequently, both signals were filtered and connected to the baseband input ports of the IQ mixers. As suggested in section II.B, the LPFs employed were the Minicircuits model VLF-2500. From Fig. 2 and section II.B, performance estimation relies on square signals arriving to the input of the low pass filters. To compensate high frequency attenuations, the emphasis functionality which modern FPGAs incorporate in analogue transceivers [29] was employed. As explained in section II.B, despite the different delays 
introduced by different IQ mixers, perfect orthogonal phase alignment can be obtained by adjusting the phases of the LOs [11]. In a back to back configuration, subcarriers were included progressively, and the phase shifters in the transmitter were adjusted ensuring maximum eye opening. After these phases had been adjusted, they remained constant and fixed for every realization of measurements. The broadband outputs of the IQ mixers were equalized in amplitude with attenuators, and then combined and amplified. Fig. 6(d) illustrates the electrical spectrum at the output of the FBMC transmitter.

The optical link was established with a polymer based OIQM [30] that presented a half-wave voltage $V_{\pi}=2.5 \mathrm{~V}$ and a bandwidth of $20 \mathrm{GHz}$. Every optical carrier was modulated generating a lower OSSB channel. A sideband suppression ratio of $20 \mathrm{~dB}$ was achieved. The optical spectrum of the WDM/OSCM signal can be observed in Fig. 6(e). The parallel MZMs inside the OIQM were biased at quadrature, and driven by the RF FBMC signal and its HT, with a total rms OMI of $\approx 20 \%$, or $\approx 500 \mathrm{mV}_{R M S}$. A sensitivity close to $-28 \mathrm{dBm}$ for a BER of $3.8 \cdot 10^{-3}$ was expected, as explained in section II.D. A higher OMI was avoided, because the peak to peak voltage signal would be higher than the $V_{\pi}$ value, and more unexpected nonlinear effects could occur in the modulator. The desired optical channel was then selected with a tunable optical band pass filter (OBPF) as shown in Fig. 6(f). The rejection ratio of the adjacent optical carrier was $22 \mathrm{~dB}$. A variable optical attenuator (VOA) was used to simulate fiber losses. The resultant signal was introduced into a preamplified optical receiver consisting of an EDFA and a photoreceiver with a $20 \mathrm{GHz}$ bandwidth. The EDFA was operated in constant power mode to ensure an average optical power of $5 \mathrm{dBm}$ was fed to the photo-receiver regardless of the average received optical power, $P_{I N}$. The electrical FBMC signal was obtained at the output of the photo-receiver, as illustrated in Fig. $6(\mathrm{~g})$, and then demodulated.

In the FBMC receiver, the incoming signal was amplified and split, before IQ mixers performed the demodulation. As suggested in section II.B, each resultant output signal was low pass filtered with two cascaded Bessel filters, $B_{2,4}(f)$. The functionality of a PLL was emulated using variable phase shifters to lock the LOs of the IQ mixers to the phase of the incoming subchannels $[17,18]$. The received baseband signals presented an optimum sampling point with minimum ISI and ICI. A real-time BER tester (BERT) was used to measure performance.

Given the close allocation of optical channels, there are two contributions that set the minimum distortion of the system. Firstly, the imperfectly suppressed data side bands from the neighboring optical SSB channel [9]. Secondly, the optical carrier of the adjacent optical channel could not be perfectly suppressed in the receiver (see Fig. 6(f)), adding cross-talk due to the beating in the photo-detector. As the transmitted baseband streams are uncorrelated, and with the selected frequency plan, it can be deduced that in all the cases both distortions are uncorrelated with the desired signal. Therefore, no further decorrelation of the WDM signal is required.

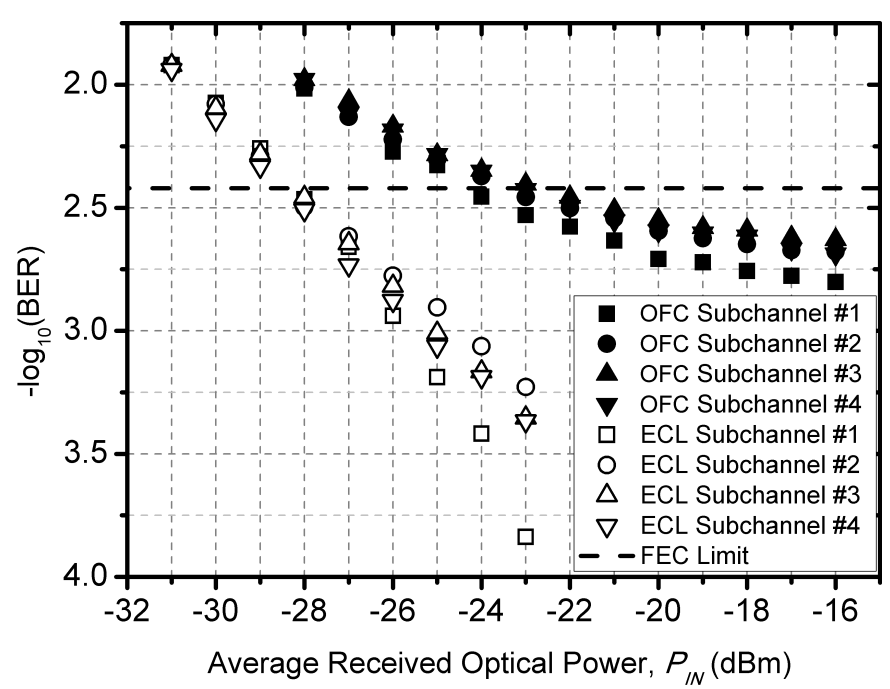

Fig. 7. Comparison of performance between the worst optical channel in the WDM signal and a single channel based on one low-RIN high-CNR ECL. FEC limit for a $7 \%$ overhead

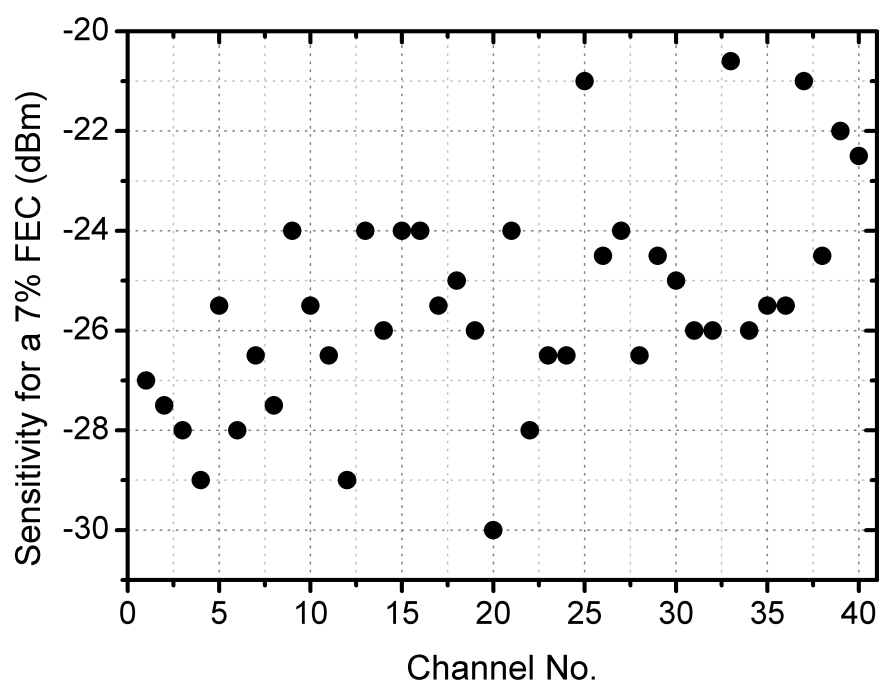

Fig. 8. Individual sensitivity for all the subchannels.

\section{B. Results and discussion}

The optical carriers were modulated generating lower OSSB subchannels (see Fig. 6(e)). As it can be observed in Fig. 6(c), the fifth optical channel presented the lowest CNR for this modulation, $\approx 50 \mathrm{~dB}$, and, accordingly, the worst performance. For this channel, Fig. 7 shows the average BER of the two components (I and Q) transmitted in every electrical subchannel, as a function of the average optical power at the input of the pre-amplified optical receiver, $P_{I N}$. Measurements were taken with an optical back to back link. The performance of all the electrical subchannels was similar and the sensitivity for the $7 \%$ HD-FEC code was $\approx-23 \mathrm{dBm}$. Under the same conditions, performance was measured using only one optical channel whose optical carrier was coming from an ECL. The RIN of the ECL was $<-140 \mathrm{~dB} / \mathrm{Hz}$, in contrast with $\approx-130 \mathrm{~dB} / \mathrm{Hz}$ in the OFC [25]. The CNR of the ECL was $>80 \mathrm{~dB}$, in contrast with the $\approx 50 \mathrm{~dB}$ in the worst case of the OFC. As it can also be observed in Fig. 7, the sensitivity achieved with the ECL was $\approx-28 \mathrm{dBm}$ for all the 


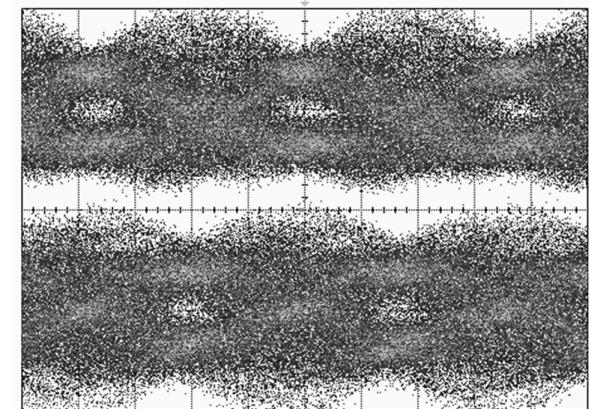

(a)

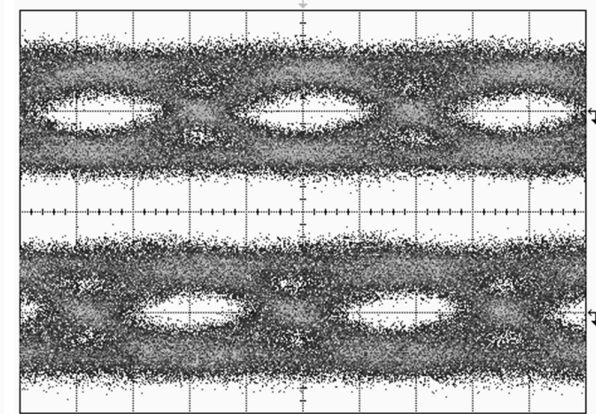

(b)

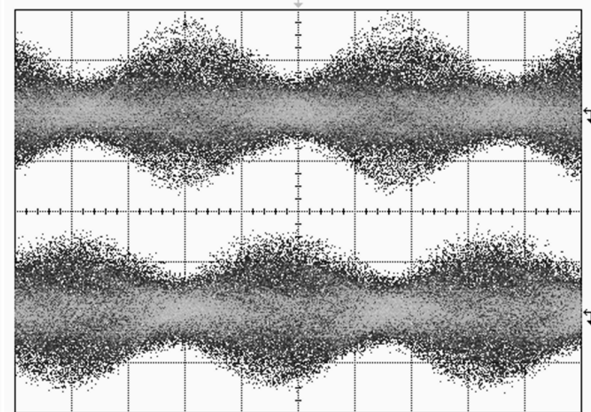

(c)

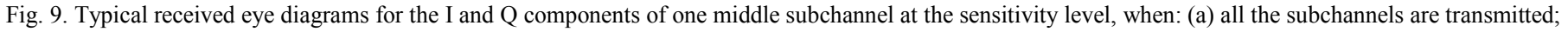
(b) only the desired subchannel is transmitted, and (c) only the non-desired subchannels are transmitted. 100 ps per time division.

electrical subchannels, similar to the best achievable value predicted in section II.D. It should be noted that in the WDM case, the optical channel 5 experienced interference from the imperfectly suppressed sideband of channel 4 [9]. It can be concluded that the maximum penalty generated by the tightly allocated WDM solution based on an externally-injected gain switched DFB laser was $5 \mathrm{~dB}$ with respect to a high performance individual channel. The penalty was due to the higher RIN and lower CNR of the OFC, and the interference generated by the adjacent optical channel. In Fig. 7, it can also be observed that the WDM case, in contrast with the ECL case, stablishes a higher noise floor that limits the best performance that can be obtained regardless the value of $P_{I N}$.

A more comprehensive approach requires individually analyzing the 40 components $(5 \times 4 \times 2,5$ optical carriers, 4 electrical subcarriers, and 2 due to the quadrature modulation on each subcarrier). Another realization of the measurements was carried out obtaining the sensitivity for a 7\% FEC for the 40 electrical components. The results are illustrated in Fig. 8. Components 1 to 8 belong to the first optical channel and components 33 to 40 belong to the fifth one. There are several points that can be discussed. Firstly, some subchannels presented a disparity between the I and Q components (up to $5.5 \mathrm{~dB}$ ). This IQ imbalance was mainly attributed to the limited performance of the electrical IQ mixers with broadband signals. Secondly, it can be confirmed that the CNR of the optical lines in the OFC determines the final performance of the optical channels. In this case the best CNR value corresponds to optical channel $1, \approx 65 \mathrm{~dB}$ (see Fig. 6 (c)), and it presents the best performance. Optical channel five presents the worst performance with a CNR of $\approx 50 \mathrm{~dB}$. Finally, all the components were able to achieve BER values below the FEC limit, with the sensitivity of the worst case at $-20.5 \mathrm{dBm}$. After 7\% FEC overheads, a net data rate higher than $100 \mathrm{Gbit} / \mathrm{s}$ was achieved. A typical eye diagram received at the sensitivity level is shown in Fig. 9 (a). It can be decomposed into the desired signal, Fig. 9 (b), plus the ICI, Fig. 9 (c). As predicted in section II.B, ICI is higher than ISI. It should be noted that, potentially, better sensitivities can be achieved by applying a level of carrier suppression in the transmitter either with optical filters [6] or low-biasing the OIQM [13].

The presented results achieve a higher spectral efficiency than any previously reported broadband real-time DSP-less WDM/SCM link, as they have made use of traditional nonorthogonal QPSK modulation [9, 31]. Neglecting the optical carriers, the spectral efficiency of the presented OSCM scheme is $1.6 \mathrm{bit} / \mathrm{s} / \mathrm{Hz}$, approaching $2 \mathrm{bit} / \mathrm{s} / \mathrm{Hz}$ for a higher number of subcarriers, substantially higher than traditional QPSK solutions that are limited to $1 \mathrm{bit} / \mathrm{s} / \mathrm{Hz}$. In addition, it should be noted that DD broadband SCM/16QAM links that have been reported always relied on DSP [32]. OSCM/QPSK can obtain a spectral efficiency closer to SCM/16-QAM without using DSP and requiring a simpler baseband receiver consisting of an electrical digital comparator.

\section{CONCLUSION}

SCM can leverage the maturity of microwave components to implement reliable real-time electro-optical transceivers. Purely analogue SCM implementations have the potential to achieve good performance in terms of power consumption, latency and tolerance to dispersion, but have been traditionally limited to low values of spectral efficiency. The previous weakness has been overcome by the transmission of orthogonal electrical subchannels following FBMC theory.

For the first time, a broadband real-time all-analogue WDM/OSCM scheme has been demonstrated, achieving a higher spectral efficiency than any previously reported equivalent link. The most relevant features of the described experiment are emphasized below. First, a net rate higher than $100 \mathrm{Gbit} / \mathrm{s}$ would be achieved after applying a 7\% FEC. Second, a gain switched laser was used to generate all the optical carriers. The stability of this type of OFC was a key parameter that allowed a tight allocation of optical channels. Third, the optical comb and the microwave scheme largely relied on standard or off-the-shelf components. Finally, the baseband rate used in the experiment was $2.7 \mathrm{Gbit} / \mathrm{s}$, directly compatible with the typical electrical interfaces employed in computing and electro-optical transceivers. The described features ensure that OSCM is a promising technique to be considered in the design of future WDM electro-optical transceivers.

\section{REFERENCES}

[1] Z. Li, I. Shubin, and X. Zhou, "Optical interconnects: recent advances and future challenges," Opt. Exp., vol. 23, pp. 3717-3720, 2015.

[2] J. C. Rasmussen, T. Takahara, T. Tanaka, Y. Kai, M. Nishihara, T. Drenski, et al., "Digital signal processing for short reach optical links," in Proc. ECOC, 2014, p. Tu.1.3.3. 
[3] A. Morea, S. Spadaro, O. Rival, J. Perello, F. Agraz, and D. Verchere, "Power management of optoelectronic interfaces for dynamic optical networks," in Proc. ECOC, 2011, p. We.8.K.3.

[4] N. Nambath, R. K. Raveendranath, D. Banerjee, A. Sharma, A. Sankar, and S. Gupta, "Analog Domain Signal Processing-Based Low-Power 100-Gb/s DP-QPSK Receiver," J. Lightw. Technol., vol. 33, pp. 31893197, 2015.

[5] T. E. Darcie, M. E. Dixon, B. L. Kasper, and C. A. Burrus, "Lightwave system using microwave subcarrier multiplexing," Electron. Lett., vol. 22, pp. 774-775, 1986.

[6] R. Hui, Z. Benyuan, H. Renxing, C. T. Allen, K. Demarest, and D. Richards, "Subcarrier multiplexing for high-speed optical transmission," J. Lightw. Technol., vol. 20, pp. 417-427, 2002.

[7] M. Salter, D. Platt, L. Pettersson, L. Aspemyr, and B. Mingquan, "Circuits and system simulations for $100 \mathrm{~Gb} / \mathrm{s}$ optical SCM transmission," in Proc. ICECS, 2009, pp. 960-963.

[8] G. H. Smith, D. Novak, and Z. Ahmed, "Overcoming chromaticdispersion effects in fiber-wireless systems incorporating external modulators," IEEE Trans. Microw. Theory Tech., vol. 45, pp. 1410$1415,1997$.

[9] F. A. Gutierrez, P. Perry, F. Smyth, A. D. Ellis, and L. P. Barry, "Impact of band rejection in multichannel broadband subcarrier multiplexing," $J$. Opt. Commun. Netw., vol. 7, pp. 248-252, 2015.

[10] F. A. Gutierrez, E. P. Martin, P. Perry, A. D. Ellis, P. Anandarajah, F. Smyth, et al., "100 Gbit/s Real-Time All-analogue Filter Bank OFDM based on a Gain-Switched Optical Comb," in Proc. ECOC, 2015, p. Th.1.5.4.

[11] F. A. Gutierrez, P. Perry, E. P. Martin, A. D. Ellis, F. Smyth, and L. P. Barry, "All-Analogue Real-Time Broadband Filter Bank Multicarrier Optical Communications System," J. Lightw. Technol., vol. 33, pp. 5073-5083, 2015.

[12] P. M. Anandarajah, Z. Rui, R. Maher, M. D. Gutierrez Pascual, F. Smyth, V. Vujicic, et al., "Flexible optical comb source for super channel systems," in Proc. OFC, 2013, p. OTh3I.8.

[13] F. A. Gutierrez, P. Perry, F. Smyth, A. D. Ellis, and L. P. Barry, "Optimum Bias Point in Broadband Subcarrier Multiplexing With Optical IQ Modulators," J. Lightw. Technol., vol. 33, pp. 258-266, 2015.

[14] B. Saltzberg, "Performance of an Efficient Parallel Data Transmission System," IEEE Trans. Commun. Tech., vol. 15, pp. 805-811, 1967.

[15] B. Farhang-Boroujeny, "OFDM Versus Filter Bank Multicarrier," IEEE Signal Process. Mag., vol. 28, pp. 92-112, 2011.

[16] H. Li, Q. Yang, C. Li, X. Zhang, C. Li, T. Jiang, et al., "Optical OFDM/OQAM for the Future Optical Communication," in Proc. ACPC, 2014, p. AW4H.5.

[17] P. Kourtessis and S. D. Walker, "A Complete 8-GHz QPSK-MODEM Featuring Novel Subcarrier and Data Synchronization for Optical Communications," IEEE Trans. Comm., vol. 55, pp. 987-995, 2007.

[18] I. Seto, H. Shoki, and S. Ohshima, "Optical subcarrier multiplexing transmission for base station with adaptive array antenna," IEEE Trans. Microw. Theory Tech., vol. 49, pp. 2036-2041, 2001.

[19] J. Armstrong, "OFDM for Optical Communications," J. Lightw. Technol., vol. 27, pp. 189-204, 2009.

[20] E. Sooudi, S. Sygletos, A. D. Ellis, G. Huyet, J. G. McInerney, F. Lelarge, et al., "Optical Frequency Comb Generation Using Dual-Mode Injection-Locking of Quantum-Dash Mode-Locked Lasers: Properties and Applications," J. Sel. Top. Quantum Electron., vol. 48, pp. 13271338, 2012.

[21] G. T. Harvey and L. F. Mollenauer, "Harmonically mode-locked fiber ring laser with an internal Fabry-Perot stabilizer for soliton transmission," Opt. Lett., vol. 18, pp. 107-109, 1993.

[22] R. Wu, V. R. Supradeepa, C. M. Long, D. E. Leaird, and A. M. Weiner, "Generation of very flat optical frequency combs from continuous-wave lasers using cascaded intensity and phase modulators driven by tailored radio frequency waveforms," Opt. Lett., vol. 35, pp. 3234-3236, 2010.

[23] T. Sakamoto, T. Kawanishi, and M. Izutsu, "Widely wavelength-tunable ultra-flat frequency comb generation using conventional dual-drive Mach-Zehnder modulator," Electon. Lett., vol. 43, pp. 1039-1040, 2007.

[24] P. M. Anandarajah, R. Maher, Y. Q. Xu, S. Latkowski, J. O'Carroll, S. G. Murdoch, et al., "Generation of Coherent Multicarrier Signals by Gain Switching of Discrete Mode Lasers," Photon. J., vol. 3, pp. 112$122,2011$.

[25] R. Zhou, S. Latkowski, J. O’Carroll, R. Phelan, L. P. Barry, and P. Anandarajah, "40nm wavelength tunable gain-switched optical comb source," Opt. Exp., vol. 19, pp. B415-B420, 2011.
[26] V. Vujicic, P. M. Anandarajah, R. Zhou, C. Browning, and L. P. Barry, "Performance Investigation of IM/DD Compatible SSB-OFDM Systems Based on Optical Multicarrier Sources," Photon. J., vol. 6, pp. 1-10, 2014.

[27] "Forward Error Correction for High Bit-Rate DWDM Submarine Systems," ITU-T Recommendation G.975.1, 2004.

[28] B. Olsson, J. Martensson, A. Kristiansson, and A. Alping, "RF-assisted optical dual-carrier $112 \mathrm{Gbit} / \mathrm{s}$ polarization-multiplexed 16-QAM transmitter," in Proc. OFC, 2010, p. OMK5.

[29] "Virtex 6 FPGA GTX Transceivers: User Guide.," ed: Xilinx, 2011.

[30] Y. Guomin, E. Miller, J. Mallari, W. Cailin, C. Baoquan, C. Hui, et al., "Small form factor thin film polymer modulators for telecom applications," in Proc. OFC, 2012, p. OM3J.1.

[31] E. Rochat, P. Kourtessis, M. Webster, T. Quinlan, S. Dudley, S. D. Walker, et al., "Ultra-High Capacity Transmission over $3 \mathrm{~km}$ of Legacy 50 um-Multimode-Fibre using C-Band HDWDM and QuadratureSubcarrier Multiplexing," in Proc. ECOC, 2002, p. 8.2.2.

[32] B. Olsson, A. Kristiansson, and A. Alping, "40 Gbit/s, 16-QAM, transmission utilizing electronic sub-carrier technique and direct detection reception," in Proc. ACP, 2009, p. ThC3. 